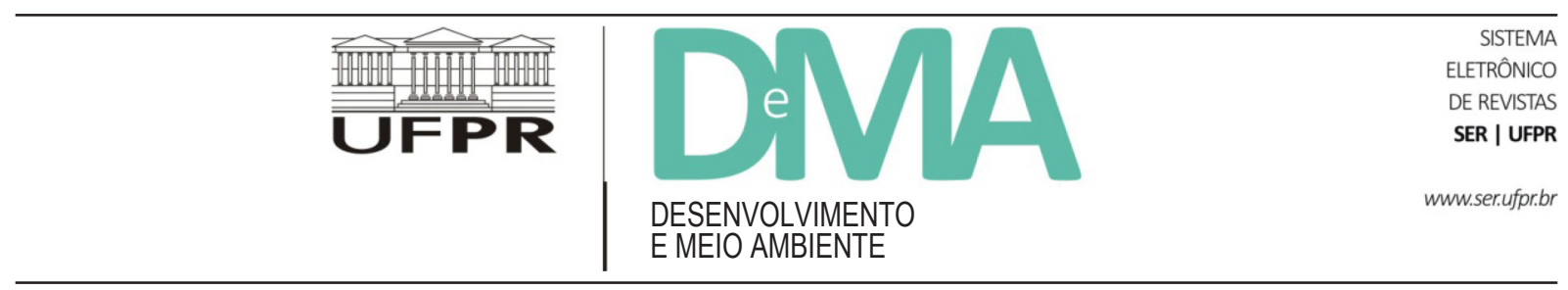

\title{
Animais silvestres utilizados como recurso alimentar em assentamentos rurais no município de Uruará, Pará, Brasil
}

\section{Wild Animals Used as Food Resource in Rural Settlements in the Municipality of Uruará, Pará, Brazil}

\author{
Reinaldo Lucas CAJAIBA ${ }^{1 *}$, Wully Barreto da SILVA², Paulo Ricardo R. PIOVESAN ${ }^{3}$ \\ ${ }^{1}$ Universidade de Trás-os-Montes e Alto Douro (UTAD), Vila Real, Portugal. \\ ${ }^{2}$ Universidade Federal do Pará (UFPA), Altamira, PA, Brasil. \\ ${ }^{3}$ Instituto Nacional de Pesquisas da Amazônia (INPA), Manaus, AM, Brasil. \\ *E-mail de contato: reinaldocajaiba@hotmail.com
}

Artigo recebido em 8 de dezembro de 2014, versão final aceita em 19 de junho de 2015.

RESUMO O consumo de animais cinegéticos tem importância fundamental para a alimentação humana em diferentes áreas tropicais. Conhecer as espécies escolhidas, as técnicas de capturas e a quantidade escolhida são aspectos fundamentais para compreender a forma de uso e o grau de ameaça da caça sobre cada espécie silvestre. Nessa perspectiva, o presente trabalho teve como objetivo registrar as principais espécies de animais silvestres utilizados como recurso alimentar em cinco assentamentos rurais no município de Uruará, Pará, além de qualificar as principais técnicas de captura dessas espécies. A obtenção dos dados ocorreu através de entrevistas semiestruturada e observação participante. Para a seleção dos entrevistados, adotou-se o método "bola de neve". Como resultado, identificaram-se 38 espécies de animais consumidas, sendo a maioria delas mamíferos (42,1\%), seguidos por aves $(39,4 \%)$ e répteis (18,5\%). As espécies mais citadas foram: Cuniculus paca $(\mathrm{n}=156)$, Euphractus sexcinctus $(\mathrm{n}=154)$ e Pecari tajacu $(\mathrm{n}=137)$. Quanto aos métodos de caça, o uso da espingarda é a técnica mais frequente. Entre os animais citados Priodontes maximus, Tayassu pecari, Tapirus terrestris, Myrmecophaga tridactyla, Podocnemis unifilis, Tinamus tao, Crax fasciolata e Podocnemis unifilis figuram na lista de espécies ameaçadas. Os resultados apontam a necessidade urgente de programas educativos com os agricultores quanto ao uso insustentável dos animais.

Palavras-chave: Amazônia; animais cinegéticos; etnozoologia.

ABSTRACT The consumption of hunting animals is of fundamental importance for human subsistence in different tropical areas. Knowing the chosen species, the techniques of capture and the quantity are fundamental aspects to understand how to use and the degree of threat of hunting on each wild species. In this perspective, the objective of this work was to record the wild animal species most commonly used as food resource in five rural settlements in the municipality of Uruará-PA, in addition to qualify the main techniques of capturing these species. The data collection was made through semi-structured interviews and participant observation. 
For the selection of the interviewees, we adopted the "snowball" method. As a result, we identified 38 species of animals consumed, most being mammals (42.1\%), followed by birds $(39.4 \%)$ and reptiles $(18.5 \%)$. The species most frequently mentioned were: Cuniculus paca $(\mathrm{n}=156)$, Euphractus sexcinctus $(\mathrm{n}=154)$ and Pecari tajacu $(\mathrm{n}=137)$. The most used technique was hunting with shotgun. Among the animals cited, Priodontes maximus, Tayassu pecari, Tapirus terrestris, Myrmecophaga tridactyla, Podocnemis unifilis, Tinamus tao, Crax fasciolata and Podocnemis unifilis are in the list of threatened species. The results point to the urgent need for educational programs to farmers regarding the unsustainable use of animals.

Keywords: Amazon; hunting animals; ethnozology.

\section{Introdução}

O homem estabeleceu relações com a fauna desde tempos remotos, representando uma das mais antigas formas de interação entre humanos e a biodiversidade (Alves \& Souto, 2010). Dentre essas interações, a caça é uma das mais antigas atividades humanas de que se tem conhecimento (Alves et al., 2010). Esse tipo de atividade foi fundamental para o melhoramento da aquisição proteica, vestimenta, controle de predadores e cura de doenças (Fernandes-Ferreira, 2010; Vasconcelos Neto et al., 2012).

A captura de animais silvestres para alimentação é fundamental para a subsistência da população humana em diferentes áreas tropicais, principalmente as que vivem em locais isolados (Davies, 2002; Figueira et al., 2003). A carne de animais silvestres apresenta alto teor proteico se comparado a outros alimentos, consumidos por essas comunidades, tais como a farinha de mandioca e o peixe (Rezende \& Schiavetti, 2010). Entretanto, a utilização frequente e constante de animais silvestres para consumo humano nessas áreas vem sendo apontada como uma das causas de extinção ou declínio populacional de várias espécies; diminuição da densidade populacional das espécies caçadas; redução da massa corporal média das populações em consequência da seleção dos animais maiores e diminuição da produtividade futura das populações caçadas (Thiollay, 2005; Thoisy et al., 2005). Ademais, ressalta-se que a caça tem seus efeitos intensificados pela fragmentação de habitat que amplia as possibilidades de acesso dos caçadores a áreas anteriormente quase inacessíveis e diminui a área de ocupação das espécies (Peres, 2001; Baía Júnior, 2006).

A atividade de captura de recursos faunísticos emprega uma diversidade de técnicas e estratégias que têm evoluído ao longo do tempo, como evidenciado em trabalhos de Ramires \& Barrella (2003) e Alves et al. (2010). Essa variedade de técnicas está relacionada à diversidade e à ecologia das presas, fatores abióticos, sazonalidade, entre outros (Alves et al., 2009; Bezerra et al., 2012).

Conhecer as espécies escolhidas, a forma de captura, a quantidade e o motivo da extração são aspectos fundamentais para compreender a forma de uso e o grau de ameaça da caça sobre cada espécie silvestre (Trinca, 2004). Nessa perspectiva, esta pesquisa teve como objetivo verificar as principais espécies de animais silvestres utilizadas como recurso alimentar, procurando também qualificar as principais técnicas de captura dessas espécies por moradores de áreas localizadas em assentamentos rurais no município de Uruará, estado do Pará, Brasil.

\section{Material e métodos}

\subsection{Local de estudo}

O estudo foi desenvolvido em cinco projetos de assentamentos da reforma agrária, localizados no município de Uruará, Pará: Rio do Peixe, Trairão, Tutuí Norte, Tutuí Sul e Uirapuru (Figura 1). A atividade agrícola dos assentados se baseia em lavouras de subsistência, tais como: mandioca (Manihot esculenta Crantz), arroz (Oryza sativa L.), batata-doce (Ipomoea batatas L.), feijão (Phaseolus sp), abóbora (Cucurbita sp) e milho (Zea mays L.). Como complemento de sua alimentação, fazem o uso de produtos oriundos da caça e da pesca.

A maioria dos produtores familiares dos assentamentos estudados produz alimentos para autoconsumo, e quando ocorre excedente de produção os destinam 
à venda. Esses produtos comercializados têm grande importância no abastecimento do mercado local, em particular nas feiras livres do município, além de ser uma forma complementar em sua renda familiar, pois o dinheiro resultante desse comércio é destinado à compra de mercadorias que eles não conseguem produzir (sal, fósforo, alguns medicamentos, roupas, entre outros).
O município de Uruará está situado no estado do Pará na Amazônia Oriental brasileira, na rodovia Transamazônica (BR-230), distando aproximadamente $1.000 \mathrm{~km}$ da capital Belém (Figura 1). A população do município é de 44.789 habitantes, sendo que 45,45\% residem no meio rural (IBGE, 2010).

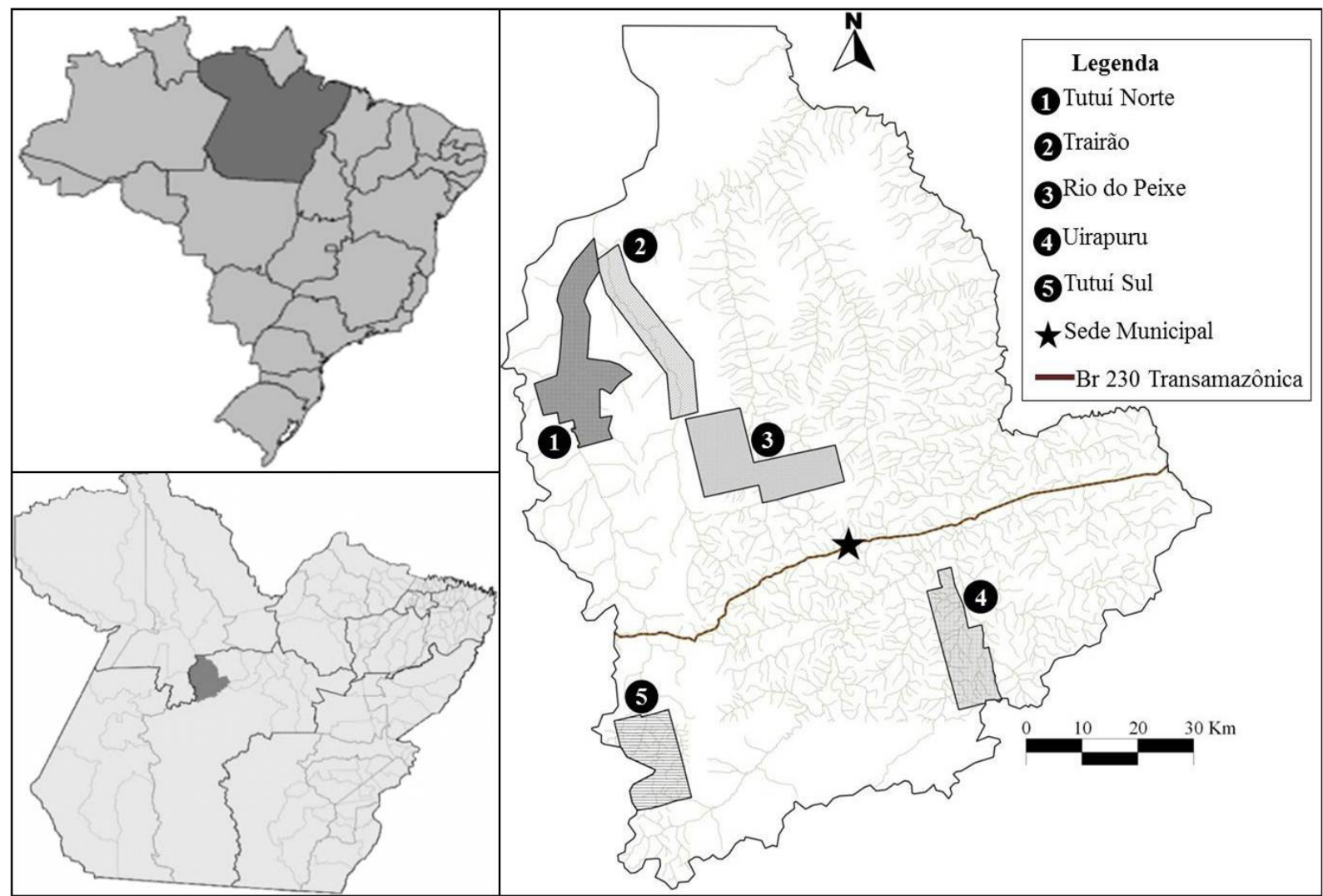

FIGURA 1 - Localização dos assentamentos estudados no município de Uruará, estado do Pará, Brasil.

\subsection{Coleta de dados}

O trabalho de campo foi realizado entre agosto de 2013 e julho de 2014. A obtenção das informações ocorreu por meio de entrevistas semiestruturadas, compreendendo a coleta de dados através de perguntas formuladas, porém com respostas livres sobre o tema proposto, e de observações diretas durante as entrevistas.

Os informantes foram escolhidos pela técnica "Bola de Neve" (Biernacki \& Waldorf, 1981), que consiste em localizar os demais informantes-alvo da pesquisa a partir da indicação dos primeiros. Essa 
sequência de indicação possibilitou o reconhecimento de informantes-chave (Nardel, 1939), que são aquelas pessoas reconhecidas na comunidade por possuir maior experiência ou deter maior conhecimento sobre determinado assunto de interesse da pesquisa. Definimos essas pessoas como os presidentes das associações existentes em cada assentamento rural e/ou aquelas que residiam maior tempo nesse local. Como forma complementar às entrevistas, foi realizada o percurso guiado em campo (Marques, 1995), em que o chefe da residência serviu de guia em áreas ou nas atividades que foram desenvolvidas. Essa técnica é necessária para validar e/ou complementar informações recebidas dos informantes durante as entrevistas e foi utilizada para identificar os locais de caça informados pelos caçadores (Rezende \& Schiavetti, 2010).

Foram entrevistadas somente pessoas com idade igual ou superior a 18 anos e, prioritariamente, os principais responsáveis pelos domicílios pesquisados. As entrevistas foram gravadas com aparelho MP3, sendo transcritas com a máxima fidelidade possível e organizadas em um banco de dados padronizado.

A fim de determinar o perfil dos entrevistados, foram relacionadas questões contendo informações de caráter socioeconômico com as seguintes variáveis: sexo, idade, escolaridade, número de pessoas residentes no domicílio e renda familiar. Por fim, com o objetivo de determinar a relação dos agricultores com o consumo de animais silvestres, foram investigados a frequência e locais de consumo, principais espécies consumidas, formas e locais de captura.

\subsection{Análise dos dados}

Os resultados foram analisados qualitativamente, segundo o modelo de união das diversas competências individuais (Marques, 1991). Segundo este modelo, todas as informações referentes ao assunto pesquisado são consideradas (Rezende \& Schiavetti, 2010). Quando houve a necessidade de confirmar a consistência e a veracidade de determinadas respostas, recorreu-se às entrevistas repetidas em situações sincrônicas e diacrônicas (Marques, 1991).
Para verificar a similaridade dos animais consumidos pelos agricultores de cada assentamento, aplicou-se o teste de similaridade de Jaccard. Utilizou-se o teste $\mathrm{H}$ de Kruskal-Wallis para verificar se há diferença do número de espécies utilizadas de acordo com as variáveis idade, renda e escolaridade. Já a correlação de Spearman foi aplicada para verificar se o número de indivíduos por família influencia no número de animais consumidos. O nível de significância para todos os testes foi de $5 \%(\mathrm{p}<0,05)$.

Para cada espécie de animal citada foi calculado seu respectivo valor de uso (VU) (adaptado da proposta de Phillips et al., 1994), o que possibilitou demonstrar a importância relativa da espécie conhecida localmente, independentemente da opinião do pesquisador (Nobrega et al., 2011). O Valor de Uso foi calculado através da fórmula $\mathrm{VU}=\Sigma \mathrm{U} / \mathrm{n}$; em que $\mathrm{VU}=$ valor de uso da espécie, $\mathrm{U}=$ número de citações por espécie, $\mathrm{n}=$ número de informantes.

\section{Resultados}

O número de domicílios entrevistados foi 34 nos assentamentos Tutuí Norte e Trairão, 32, em Uirapuru e Tutuí Sul, e 31, em Rio do Peixe, totalizando 163 unidades familiares, sendo que todos os entrevistados eram chefes dos domicílios. A idade dos participantes variou entre 23 a 69 anos, sendo $88 \%$ do sexo masculino e $12 \%$ do sexo feminino. Quanto à escolaridade dos entrevistados, a maioria (43\%) era analfabeta; 34\% eram semianalfabetos, ou seja, sabiam assinar apenas o nome; $13 \%$ tinham o ensino fundamental inferior ( $5^{\mathrm{a}}$ série); $6 \%$, o ensino fundamental completo; e apenas $4 \%$ tinham o ensino médio completo. Foi possível verificar in loco que a maioria dos entrevistados é de outros estados, principalmente do Nordeste, que vieram para o município em busca de terra farta, barata e produtiva.

A fauna cinegética registrada para os assentamentos estudados foi representada por 38 espécies distribuídas em 25 famílias. Dentre as espécies citadas, os mamíferos apresentaram maior riqueza taxonômica $(\mathrm{n}=$ $16 / 42,1 \%)$, seguida por aves $(n=15 / 39,4 \%)$ e répteis ( $n$ $=7 / 18,5 \%$ ). Para os mamíferos, as famílias com maior número de espécies citadas foram Myrmecophagidae, 
Dasyproctidae, Cervidae, Tayassuidae, Dasypodidae ( $n=2$ espécies cada); entre as aves, Cracidae $(n=4$ espécies), Tinamidae ( $n=3$ espécies) e Psittacidae ( $n=$ 2 espécies); e entre os répteis, Testudinidae, com duas espécies (Tabela 1). Os cinco assentamentos apresentaram em comum 22 espécies de animais cinegéticos, o que equivale a $56,4 \%$ do total de espécies citadas. A Tabela 2 representa a similaridade dos principais tipos de caça entre os assentamentos. As comunidades agruparam-se de acordo com as espécies de valor cinegético mais utilizadas pelos agricultores. Os assentamentos Tutuí Sul e Trairão apresentaram maior similaridade quanto aos animais caçados (Coeficiente Jaccard $=0,84$ ), enquanto os assentamentos Tutuí Norte e Uirapuru apresentaram menor similaridade (Coeficiente Jaccard $=0,56$ ). Vale ressaltar que, em todos os assentamentos, a paca, o tatu-peba e o caititu foram os animais silvestres mais citados.

TABELA 1 - Espécies de animais silvestres utilizadas como alimento em cinco assentamentos rurais no município de Uruará, Pará. TN = Tutuí Norte, TS = Tutuí Sul, Ui = Uirapuru, RP = Rio do Peixe, Tr = Trairão, VU = Valor de Uso.

\begin{tabular}{|c|c|c|c|c|c|c|c|c|}
\hline \multirow{2}{*}{ Classe/ Família/ Nome científico } & \multirow{2}{*}{ Nome local } & \multicolumn{5}{|c|}{ Assentamentos (n. citações) } & \multirow{2}{*}{$\mathbf{V U}$} & \multirow{2}{*}{ Total } \\
\hline & & $\mathbf{T N}$ & TS & Ui & $\mathbf{R P}$ & $\operatorname{Tr}$ & & \\
\hline \multicolumn{9}{|l|}{ MAMMALIA } \\
\hline \multicolumn{9}{|l|}{ Bradypodidae } \\
\hline Bradypus variegatus (Schinz, 1825) & Preguiça & - & - & 1 & - & 1 & 0,01 & 2 \\
\hline \multicolumn{9}{|l|}{ Atelidae } \\
\hline Alouatta spp. & Macaco guariba & 1 & - & - & 1 & - & 0,01 & 2 \\
\hline \multicolumn{9}{|l|}{ Dasypodidae } \\
\hline Priodontes maximus (Kerr, 1792) & Tatu-canastra & 2 & 19 & 10 & 4 & 6 & 0,25 & 41 \\
\hline Euphractus sexcinctus (Linnaeus, 1758) & Tatu-peba & 32 & 31 & 30 & 32 & 29 & 0,94 & 154 \\
\hline \multicolumn{9}{|l|}{ Didelphidae } \\
\hline Didelphis spp. & Mucura, saruê, gambá & - & 4 & 4 & 2 & 5 & 0,09 & 15 \\
\hline \multicolumn{9}{|l|}{ Tayassuidae } \\
\hline Pecari tajacu (Linnaeus, 1758) & Caititu, cateto & 29 & 31 & 26 & 23 & 28 & 0,84 & 137 \\
\hline \multicolumn{9}{|l|}{ Hydrochaeridae } \\
\hline $\begin{array}{l}\text { Hydrochoerus hydrochoeris (Linnaeus, } \\
1766 \text { ) }\end{array}$ & Capivara & 22 & 21 & 19 & 23 & 27 & 0,68 & 112 \\
\hline \multicolumn{9}{|l|}{ Tapiridae } \\
\hline Tapirus terrestris (Linnaeus, 1758) & Anta & 19 & 14 & 20 & 18 & 18 & 0,54 & 89 \\
\hline \multicolumn{9}{|l|}{ Cervidae } \\
\hline Mazama americana & Veado-mateiro & 14 & 9 & 13 & 13 & 10 & 0,36 & 59 \\
\hline Mazama nemoviraga & Fuboca & 29 & 17 & 15 & 25 & 24 & 0,67 & 110 \\
\hline
\end{tabular}


Dasyproctidae

Cuniculus paca (Linnaeus, 1766)

Dasyprocta spp.

Myrmecophagidae

Myrmecophaga tridactyla (Linnaeus, 1758)

Tamandua tetradactyla (Linnaeus, 1758)

Leporidae

Sylvilagus brasiliensis (Linnaeus, 1758)

AVES

Columbidae

Leptotila spp.

Columbina spp.

Rallidae

Rallidae sp.

Tinamidae

Tinamus guttatus (Pelzeln, 1863)

Tinamus tao (Temminck, 1815)

Crypturellus spp.

Cariamidae

Cariama cristata (Linnaeus, 1766)

Cracidae

Penelope spp1.

Penelope spp2.

Crax fasciolata (Spix, 1825)

Mitu tuberosa (Spix, 1825)

$\begin{array}{llllllll}\text { Paca } & 34 & 31 & 30 & 29 & 32 & 0,95 & 156 \\ \text { Cutia } & 23 & 25 & 25 & 23 & 21 & 0,71 & 117\end{array}$

Tamanduá-bandeira

Tamanduá pequeno

Coelho-do- mato, tapiti

$-$

1

2

$-\quad 0,01$

3 
Anatidae

Cairina moschata (Linnaeus, 1758)

Ramphastidae

Ramphastos spp.

Psittacidae

Ara spp.

Amazona spp.

REPTILIA

Teiidae

Tupinambis spp.

Téju, teiú

Camaleão, lagarto-

-verde

Testudinidae

Chelonoidis spp.

Pelomedusidae

Podocnemis expansa (Schweigger, 1812)

Podocnemis unifilis (Troschel, 1848)

Alligatoridae

Melanosuchus niger (Spix, 1825)

Boidae

Boa constrictor (Forcart, 1960)
Jabuti

Tartaruga

Tracajá

Jacaré-açu

Jiboia
Pato-do-mato, pato-selvagem

Tucano

Arara

Papagaio

1

$-$

2

4

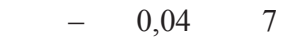

11

$\begin{array}{llllll}5 & 8 & - & 9 & 1 & 0,14\end{array}$

$\begin{array}{llllll}10 & 8 & 9 & 10 & 6 & 0,26\end{array}$

$\begin{array}{lllllll}6 & - & 2 & 5 & 1 & 0,08 & 14\end{array}$

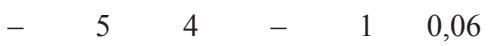
10 
TABELA 2 - Matriz do índice de similaridade de Jaccard referente aos animais silvestres consumidos nos cinco assentamentos estudados. TN = Tutuí Norte; TS = Tutuí Sul; Ui = Uirapuru; RP = Rio do Peixe; $\operatorname{Tr}=$ Trairão.

\begin{tabular}{cccccc}
\hline $\begin{array}{c}\text { Assenta- } \\
\text { mentos }\end{array}$ & TN & TS & Ui & RP & Tr \\
\hline TN & 1 & - & - & - & - \\
TS & 0,64 & 1 & - & - & - \\
Ui & 0,56 & 0,73 & 1 & - & - \\
RP & 0,82 & 0,78 & 0,68 & 1 & - \\
$\operatorname{Tr}$ & 0,71 & 0,84 & 0,74 & 0,81 & 1 \\
\hline
\end{tabular}

Os Valores de Uso (VU) calculados variaram de 0,01 a 0,95 (Tabela 1). Alguns táxons apresentaram VU acima de 0,5, como as aves rolinha (Columbina spp.; VU = 0,61), jacumirim (Penelope spp2.; VU = 0,61), inhambu (Crypturellus spp.; VU =0,59), jacu (Penelope spp.1; VU =0,58) e pato-do-mato (Cairina moschata; $\mathrm{VU}=0,53)$. Dentre os mamíferos, paca (Cuniculus paca; VU =0,95), tatu-peba (Euphractus sexcinctus; VU =0,94), capivara (Hydrochoerus hydrochoeris; $\mathrm{VU}=0,68)$, anta (Tapirus terrestris; $\mathrm{VU}=$ 0,54), veado-fuboca (Mazama nemoviraga; $\mathrm{VU}=0,67$ ) e cutia (Dasyprocta spp.; VU $=0,71$ ) apresentaram os maiores valores de uso, enquanto o jabuti (Chelonoidis spp.; $\mathrm{VU}=0,57$ ) foi o único do grupo dos répteis com VU acima de 0,5 .

Não foi observada diferença significativa entre o número de espécies citadas em relação a idade e renda familiar mensal dos entrevistados $(p>0,05)$. Também não houve diferença entre o número de espécies mencionadas em relação ao nível de escolaridade $(p>0,05)$. Embora o uso de animais silvestres não difira de modo estatisticamente significante entre as escolaridades, observamos maior tendência de consumo de animais por famílias em que o entrevistado era analfabeto.

Os resultados apontam que famílias com menor número de membros (até quatro pessoas por família) tendem a capturar mais animais que famílias compostas por mais de cinco membros, sendo que a correlação negativa entre as variáveis "número de pessoas por família" e "número de animais utilizados" foi estatisticamente significativa $\left(\mathrm{r}_{\mathrm{s}}=0.2133, \mathrm{p}=0,02\right)$.

Em relação aos locais de caça, $68 \%$ dos entrevistados afirmaram caçar em matas altas ou próximo a locais com água, como rios, igarapés e lagos/lagoas. Esses locais, segundo os informantes, são frequentados por diversos animais, devido à disponibilidade de recurso alimentar. Uma pequena parte dos entrevistados (7\%) informou que utilizam as lavouras cultivadas, como milho, arroz e mandioca, para procurar os animais. Segundo eles, esses locais são ideais para capturar principalmente aves e alguns mamíferos, como caititu e cutia, que lá buscam alimentos.

Quanto ao método de caça, 71\% dos entrevistados utilizam a espingarda, sendo a espera (muntá) o meio mais eficiente para utilizar esse tipo de equipamento. O uso de arma de fogo é mais frequente para caçar animais de grande porte, como mamíferos, por exemplo; $13 \%$ dos entrevistados também fazem uso de cachorro para procura de alguns animais, como veado (mateiro) e caititu. Para a captura de aves que forrageiam o solo, a técnica mais utilizada é a arapuca. Entretanto, essas técnicas podem ser empregadas de maneira individual ou combinadas.

O tempo de permanência na atividade de caça varia conforme a disponibilidade de tempo para sua execução ou a disponibilidade de animais. Em média, os entrevistados permanecem de três a quatro horas exercendo essa atividade, principalmente aos finais de semana.

\section{Discussão}

Os resultados obtidos mostraram que o uso de animais silvestres como fonte alimentar ainda é um importante recurso utilizado para a manutenção das comunidades estudadas, como consequência da falta de condições financeiras para a compra de carne vermelha oriunda de criações de animais domésticos (Costa-Neto, 2000). Esses recursos são considerados uma fonte de proteína fundamental para diversas populações huma- 
nas que vivem na Amazônia, desde povos indígenas (Leeuwenberg \& Robinson, 1999), colonos (Ayres \& Ayres, 1979), população ribeirinhas e extrativistas (Calouro, 1995).

De acordo com Barbosa et al. (2010), a captura de animais silvestres é devida à necessidade de acrescentar na alimentação e na renda das famílias que possuem uma quantidade relativamente alta de membros. Entretanto, verificou-se que há um decréscimo na quantidade de animais capturados quando o número de membros da família aumenta, corroborando estudos de Alves et al. (2012) e Pessoa et al. (2013).

Quando analisamos as espécies de animais cinegéticas referidas neste estudo, observa-se que a maioria também foi registrada em outros estudos etnozoológicos (Alves et al., 2009; Pereira \& Schiavetti, 2010; Alves et al., 2012). O fato dos agricultores terem citado maior quantidade de mamíferos pode estar relacionado à disponibilidade e/ou preferência por esses animais, bem como aos benefícios energéticos, pois eles são usados do ponto de vista tanto alimentar quanto utilitário (fornecem matéria-prima para a elaboração de remédios e pode-se aproveitar o couro) (Rezende \& Schiavetti, 2010).

$O$ padrão de caça a vertebrados cinegéticos para uso alimentar, com preferência por mamíferos (com maior biomassa) e aves (com maior riqueza de espécies), indica que a escolha das espécies pode ser localmente influenciada pela disponibilidade, riqueza e porte das espécies-alvo, como sugerido por Alves et al. (2012). A preferência cinegética por mamíferos silvestres reflete uma tendência registrada em diferentes localidades (Dantas-Aguiar et al., 2011; Alves et al., 2012). O maior porte dos mamíferos, que implica maior retorno em biomassa para os caçadores, explica essa situação (Peres \& Nascimento, 2006; Alves et al., 2012). Segundo Redford \& Robinson (1987), geralmente os caçadores capturam para fins alimentícios mais mamíferos que aves e mais aves que répteis. Em uma pesquisa realizada no estado do Amazonas, Souza-Mazurek et al. (2000) relataram que os mamíferos representam $91 \%$ do peso total das espécies mais consumidas.

O tatu, um dos animais mais consumidos nos assentamentos, é uma espécie generalista, ou seja, tem uma dieta variada, propiciando que esse animal habite uma ampla variedade de ambientes (Medri, 2008), podendo ser encontrado também em áreas de vegetação aberta ou mata secundária, as quais ocorrem na região devido ao processo recente de desmatamento (Costa et al., 2005; Rezende \& Schiavetti, 2010). A paca, por ser o animal mais apreciado em todos os assentamentos pesquisados, devido ao sabor de sua carne, é uma espécie historicamente caçada (Rezende \& Schiavetti, 2010). Os resultados obtidos estão de acordo com Wetterberg et al. (1976) e Bonaudo et al. (2004), em que as espécies mais consumidas são: Cuniculus paca, Euphractus sexcinctus, Pecari tajacu, Dasyprocta spp., Hydrochoerus hydrochoeris, Mazama nemoviraga, Penelope spp.2 e Columbina spp.

Analisando os métodos de caça, observa-se que os agricultores empregam diversas técnicas específicas para cada tipo de presa e de habitat, sendo a espingarda a técnica mais frequente. Segundo Trinca \& Ferrari (2006), a arma de fogo confere uma caça muito mais eficaz quando comparada às armadilhas. Alves et al. (2009) relatam que a espingarda é uma ferramenta muito comum entre os caçadores, inclusive para defesa pessoal no caso de ameaças inesperadas durante a caçada.

De acordo com Vasconcelos Neto et al. (2012), a caça utilizando cães é útil particularmente para a captura de mamíferos, pois envolve o treinamento do cão para o desempenho da atividade. Redford \& Robinson (1987) afirmam que o uso de cachorros domésticos em atividades cinegéticas resulta geralmente em aumento do número de presas, embora o objetivo inicial seja a captura de um animal que servisse como alimento, o que caracteriza a caça de subsistência; entretanto, o resultado da caçada com cães se torna predatório.

Dentre os vertebrados silvestres registrados, oito espécies figuram na lista de espécies ameaçadas da International Union for Conservation of Nature (IUCN, 2014), na categoria vulnerável a extinção: Priodontes maximus, Tayassu pecari, Tapirus terrestris, Myrmecophaga tridactyla, Podocnemis unifilis, Tinamus tao, Crax fasciolata, Podocnemis unifilis. Quatro espécies registradas nas entrevistas estão em baixo risco de extinção, sendo duas delas (Podocnemis expansa e Melanosuchus niger) dependentes de medidas de conservação, e outras duas (Cuniculus paca e Tinamus guttatus) consideradas como quase ameaçadas. 
A inclusão das espécies cinegéticas registradas em listas de ameaça de extinção nos coloca diante do desafio de buscar formas de exploração que minimizem o impacto sobre as espécies cinegéticas, e, para isso, torna-se necessário compreender o contexto multidimensional que envolve as práticas cinegéticas (Alves et al., 2012).

De acordo com Ribeiro et al. (2007), a ação antrópica, caracterizada especialmente pela caça e pelo desmatamento, exerce efeitos variados sobre as densidades das espécies animais, podendo levá-las a extinção. A destruição dos ambientes naturais é a principal ameaça às comunidades de vertebrados de médio e grande porte. Porém, a caça intensa em uma região pode ter impacto bastante negativo, ocasionando a diminuição da densidade populacional das espécies e podendo alterar a dinâmica do ecossistema (Fragoso, 1991). De acordo com Leal et al. (2005), a atividade humana não sustentável, como agricultura de corte e queima, e a contínua remoção da vegetação para a criação de bovinos e caprinos têm causado o empobrecimento ambiental em larga escala. Como tal, a caça de animais selvagens deve ser considerada ao lado de outras pressões antrópicas (Alves et al., 2012).

Alves et al. (2012) citam que as principais ações a serem aplicadas para minimizar os impactos sobre as populações animais são: i) desenvolvimento de programas educacionais de manejo da vida selvagem, com fortes

\section{Referências}

Alves, R. R. N.; Gonçalves, M. B. R.; Vieira, W. L. S. Caça, uso e conservação de vertebrados no semiárido Brasileiro. Tropical Conservation Science, 5, 394-416, 2012.

Alves, R. R. N.; Mendonça, L. E. T.; Confessor, M. V. A.; Vieira, W. L. S.; Lopez, L. C. S. Hunting strategies used in the semi-arid region of northeastern Brazil. Journal of Ethnobiology and Ethnomedicine, 5, 1-16, 2009.

Alves, R. R. N.; Nougueira, E. E. G.; Araujo, H. F. P.; Brooks, S. E. Bird-keeping in the Caatinga, NE Brasil. Human Ecology, 38, 147-156, 2010. componentes de legislação ambiental e sua aplicação correta; e ii) criação de canais de comunicação entre instituições acadêmicas e governamentais com populações humanas envolvidas na caça. Além disso, medidas externas que limitem o comportamento das populações locais devem ser consideradas, tais como controlar o comércio ilegal de animais silvestres (Alves et al., 2009).

Infelizmente, os efeitos da caça sobre a fauna silvestre ainda não são fáceis de se mensurar. É necessário estimar a pressão da caça existente e os parâmetros populacionais básicos das principais espécies caçadas, para que seja possível avaliar o verdadeiro impacto sobre essas populações (Calouro, 1995; Pianca, 2004). Pesquisas futuras são sugeridas para corrigir as falhas deste trabalho, tais como identificar se há comércio ilegal de animais silvestres na região; verificar se as espécies capturadas refletem no número de indivíduos ou biomassa local; verificar se há variação sazonal do número de espécies caçadas nos assentamentos; levantar a biomassa em quilogramas de carne de animais silvestres consumidos em determinada unidade de tempo por pessoa ou unidade familiar e, com isso, analisar os efeitos da caça de subsistência sobre a população de animais silvestres consumidos na alimentação, pois, como observado por Peres (2000), o regime de caça modifica a biomassa total de vertebrados, bem como os efeitos combinados da biomassa e da densidade das espécies de diferentes classes de tamanho.

Alves, R. R. N.; Souto, W. Panorama atual, avanços e perspectivas futuras para etnozoologia no Brasil. In: Alves, R. R. N.; Souto, W. M. S.; Mourão, J. S. (Orgs.). A Etnozoologia no Brasil: importância, status atual e perspectivas. NUPEEA, Recife, p. 19-40, 2010.

Ayres, J. M.; Ayres, C. Aspectos da caça no alto Rio Aripuanã. Acta Amazonica, 9, 287-298, 1979.

Baía Júnior, P. C. Caracterização do uso comercial e de subsistência da fauna silvestre no município de Abaetetuba, PA. Dissertação (Mestrado), Universidade Federal do Pará, 2006. 
Barbosa, J. A. A.; Nobrega, V. A.; Alves, R. R. N. Aspectos da Caça e Comércio Ilegal da Avifauna Silvestre por Populações Tradicionais do Semiárido Paraibano. Revista de Biologia e Ciências da Terra, 10, 39-49, 2010.

Bezerra, D. M. M.; Araújo, H. F. P.; Alves, R. R. N. Captura de aves silvestres no semiárido brasileiro: técnicas cinegéticas e implicações para conservação. Tropical Conservation Science, 5, 50-66, 2012.

Biernacki, P. E.; Waldorf, D. Snowball sampling problems and techniques of chain referral sampling. Sociological Methods and Research, 10, 141-163, 1981.

Bonaudo, T.; Le Pendu, Y.; Albuquerque, ?. Exploração da fauna silvestre na Transamazônica. In: Sayago D.; Tourrand, J. F.; Bursztyn, M. (Eds.). Amazônia Cenas e Cenários. UNB, Brasília, Distrito Federal. 382pp, 2004.

Calouro, A. M. Caça de subsistência: sustentabilidade e padrões de uso entre seringueiros ribeirinhos e não ribeirinhos do Estado do Acre. Dissertação (Mestrado), UnB. Brasília, 1995.

Costa, L. P.; Leite, Y. L. R.; Mendes, S. L.; Ditchfield, A. D. Conservação de mamíferos no Brasil. Megadiversidade, 1, 103-112, 2005.

Costa-Neto, E. M. Conhecimento e usos tradicionais de animais por uma comunidade afro-brasileira do Parque Nacional Chapada Diamantina, Bahia, Brasil: Resultados preliminares. Interciencia, 25, 423-431, 2000.

Dantas-Aguiar, P. R.; Barreto, R. M.; Santos-Fita, D.; Santos, E. B. Hunting Activities and Wild Fauna Use: A Profile of Queixo D'antas Community, Campo Formoso, Bahia, Brazil. Bioremediation, Biodiversity and Bioavailability, 5, 1-10, 2011.

Davies, G. Bushmeat and international development. Conservation Biology, 16, 587-589, 2002.

Fernandes-Ferreira, H. Atividades cinegéticas em um Brejo de Altitude no Nordeste do Brasil: Etnozoologia e Conservação. Dissertação (Mestrado), Universidade Federal da Paraíba, 2010.

Figueira, M. L. O. A.; Carrer, C. R. O.; Silva Neto, P. B. Weight gain and evolution of a wild white-lipped peccaries under extensive and semi-extensive systems, on a Savanna area. Revista Brasileita de Zootecnia, 32, 191-199, 2003.

Fragoso, J. M. The effect of hunting on tapirs in Belize. In: Robinson, J. G.; Redford, K. H. (Eds.). Neotropical wildlife use and conservation. The University of Chicago Press, Chicago, p. 154-162, 1991.

IBGE - Instituto Brasileiro de Geografia e Estatística. Censo demográfico brasileiro. http://www.ibge.gov.br. Acesso em $01 / 10 / 2010$.

IUCN - International Union for Conservation of Nature. Red List of Threatened Species. 2014. Version 2014.2. www. iucnredlist.org

Leal, I. R.; Silva, J. M. C. D. A.; Tabarelli, M.; Lacher, T. E. Changing the Course of Biodiversity Conservation in the Caatinga of Northeastern Brazil. Conservation Biology, 19, 701-706, 2005.

Leeuwenberg, F. J.; Robinson, J. G. Traditional Management of Hunting by a Xavante Community in Central Brazil: The Search for Sustainability. In: Robinson, J. G.; Bennett, E. L. (Eds.). Hunting for sustainability in Tropical Forests (Biology and Resource Series). Columbia University Press. New York, 1999.

Marques, J. G. W. Aspectos ecológicos na Etnoictiologia dos pescadores do complexo Estuarino-Lagunar Mundaú-Manguabá Alagoas. Tese (Doutorado), Universidade Estadual de Campinas, Campinas, 1991.

Marques, J. G. W. Pescando pescadores: etnoecologia abrangente no baixo São Francisco alagoano. Universidade de São Paulo, São Paulo, 1995, 285p.

Medri, I. M. Ecologia e História Natural do Tatu-peba, Euphractus sexcinctus (Linnaeus, 1758), no Pantanal da Nhecolândia, Mato Grosso do Sul. Tese (Doutorado), Universidade de Brasília, Brasília, 2008.

Nardel, S. F. The interview technique in social anthropology. In: Barlett, F. C.; Hon, F. R. S.; Ginsberg, M.; Litt, D.; Lindgren, E. J.; Thoulesse, R. H. (Eds.). The study of society: methods and problems. Routledge \& Kegan Paul, London, p. 317-327, 1939.

Nobrega, V. A.; Barbosa, J. A. A.; Alves, R. R. N. Utilização de aves silvestres por moradores do município de Fagundes, Semiárido paraibano: uma abordagem etno-ornitológica. Sitientibus, 11, 165-175, 2011.

Pereira, J. P. R.; Schiavetti, A. Conhecimentos e usos da fauna cinegética pelos caçadores indígenas "Tupinambá de Olivença" (Bahia). Biota Neotropica, 10, 175-183, 2010.

Peres, C. A. Effects of subsistence hunting on vertebrate community in Amazonian forests. Conservation Biology, 14, 240-253. 2000. 
Peres, C. A. Synergistic effects of subsistence hunting and habitat fragmentation on Amazonian forest vertebrates. Conservation Biology, 15, 1490-1505, 2001.

Peres, C.; Nascimento, H. Impact of game hunting by the Kayapó of south-eastern Amazonia: implications for wildlife conservation in tropical forest indigenous reserves. Biodiversity and Conservation, 15, 2627-2653, 2006.

Pessoa, T. S. A.; Wagner, P. G. C.; Langguth, A. Captura e comercialização de animais silvestres no semiárido da Paraíba, Brasil, sob a perspectiva de crianças e adolescentes. Revista Nordestina de Biologia, 21, 79-100, 2013.

Phillips, O.; Gentry, A. H.; Reynel, C.; Wilki, P.; Gávez-Durand, C. B. Quantitative ethnobotany and Amazonian conservation. Conservation Biology, 8, 225-248, 1994.

Pianca, C. C. A caça e seus efeitos sobre a ocorrência de mamíferos de médio e grande porte em áreas preservadas de Mata Atlântica na Serra de Paranapiacaba, São Paulo. Dissertação (Mestrado) Universidade de São Paulo, São Paulo. 2004.

Ramires, M.; Barrella, W. Ecologia da pesca artesanal em populações caiçaras da Estação Ecológica de Juréia-Itatins, São Paulo, Brasil. Interciencia, 28, 208-213, 2003.

Redford, K.H.; Robinson, J.G. A game of choice: patterns of Indian and colonist hunting in the Neotropics. American Anthropologist, 89, 650-667, 1987.

Rezende, J. P.; Schiavetti, A. Conhecimentos e usos da fauna cinegética pelos caçadores indígenas "Tupinambá de Olivença" (Bahia). Biota Neotropica, 10, 175-183, 2010.
Ribeiro, A. S. S.; Palha, M. D. C.; Tourinho, M. M.; Whiteman, C. W.; Silva, A. S. L. Utilização dos recursos naturais por comunidades humanas do Parque Ecoturístico do Guamá, Belém, Pará. Acta Amazônica, 37, 235-240, 2007.

Souza-Mazurek, R. R. S.; Pedrinho, T.; Feliciano, X.; Hilário, W.; Gerôncio, S.; Marcelo, E. Subsistence hunting among the Waimiri Atroari Indians in central Amazônia, Brazil. Biodiversity and Conservation, 9, 579-596, 2000.

Thiollay, J. Effects of hunting on guianan forest game birds. Biodiversity and Conservation, 14, 1121-1135, 2005.

Thoisy, B.; Renoux, F.; Juliot, C. Hunting in northern French Guiana and its impacts on primate communities. Oryx, 39, 149-157, 2005.

Trinca, C. T. Caça em assentamento rural no sul da floresta amazônica. Dissertação (Mestrado), Museu Paraense Emílio Goeldi, Universidade Federal do Pará, 2004.

Trinca, C. T.; Ferrari, S. F. Caça em assentamento rural na Amazônia matogrossense. In: Jacobi, P.; Ferreira, L. C. (Org.). Diálogos em ambiente e sociedade no Brasil. Annablume, São Paulo, p. 155-167, 2006.

Vasconcelos Neto, C. F. A.; Santos, S. S.; Sousa, R. F.; Fernandes-Ferreira, H.; Lucena, R. F. P. A caça com cães (Canis lupus familiaris) em uma região do semiárido do nordeste do Brasil. Revista de Biologia e Farmácia, 1, 1-16, 2012.

Wetterberg, G. B.; Ferreira, M.; Brito, W. L.; Araújo, V. C. Espécies da fauna amazônica potencialmente preferidas para consumo nos restaurantes de Manaus. Brasil Florestal, 7 , 59-68, 1976. 